PAPER

\section{Practical multi-featured perfect absorber utilizing high conductivity silicon}

To cite this article: Abdullah Gok et al 2016 J. Opt. 18035002

View the article online for updates and enhancements.
Related content

- Ultrathin microwave metamaterial absorber utilizing embedded resistors Young Ju Kim, Ji Sub Hwang, Young Joon Yoo et al.

- Broadband, polarization-insensitive and wide-angle terahertz metamaterial absorber

Ben-Xin Wang, Ling-Ling Wang, Gui-Zhen Wang et al.

Critical coupling to Tamm plasmons Baptiste Auguié, Axel Bruchhausen and Alejandro Fainstein

\section{Recent citations}

- $\frac{\text { Photoresist template fabrication and }}{\text { template assisted growth for surface }}$
$\frac{\text { patterning of technologically important Cu }}{2 \text { ZnSnSe 4 thin films }}$
Sivasangari Sathiamoorthy et al
- Wideband 'black silicon' for mid-infrared
$\underline{\text { applications }}$
K Gorgulu et al
- High-conductivity silicon based spectrally
$\underline{\text { selective plasmonic surfaces for sensing in }}$
K Go infrared region




\title{
Practical multi-featured perfect absorber utilizing high conductivity silicon
}

\author{
Abdullah Gok ${ }^{1,2}$, Mehmet Yilmaz ${ }^{2}$, Necmi Bıyıkıı ${ }^{2,3}$, Kağan Topallı ${ }^{2,3}$ and \\ Ali K Okyay ${ }^{1,2,3}$ \\ ${ }^{1}$ Department of Electrical and Electronics Engineering, Bilkent University, Ankara, 06800 Turkey \\ ${ }^{2}$ UNAM - National Nanotechnology Research Center, Bilkent University, Ankara, 06800 Turkey \\ ${ }^{3}$ Institute of Materials Science and Nanotechnology, Bilkent University, Ankara, 06800 Turkey \\ E-mail: aokyay@ee.bilkent.edu.tr
}

Received 5 October 2015, revised 21 December 2015

Accepted for publication 14 January 2016

Published 9 February 2016

\begin{abstract}
We designed all-silicon, multi-featured band-selective perfect absorbing surfaces based on CMOS compatible processes. The center wavelength of the band-selective absorber can be varied between 2 and $22 \mu \mathrm{m}$ while a bandwidth as high as $2.5 \mu \mathrm{m}$ is demonstrated. We used a silicon-on-insulator (SOI) wafer which consists of n-type silicon ( $\mathrm{Si}$ ) device layer, silicon dioxide $\left(\mathrm{SiO}_{2}\right)$ as buried oxide layer, and n-type $\mathrm{Si}$ handle layer. The center wavelength and bandwidth can be tuned by adjusting the conductivity of the Si device and handle layers as well as the thicknesses of the device and buried oxide layers. We demonstrate proof-of-concept absorber surfaces experimentally. Such absorber surfaces are easy to microfabricate because the absorbers do not require elaborate microfabrication steps such as patterning. Due to the structural simplicity, low-cost fabrication, wide spectrum range of operation, and band properties of the perfect absorber, the proposed multi-featured perfect absorber surfaces are promising for many applications. These include sensing devices, surface enhanced infrared absorption applications, solar cells, meta-materials, frequency selective sensors and modulators.
\end{abstract}

Keywords: absorber, silicon, conductivity, resonance, absorption

(Some figures may appear in colour only in the online journal)

\section{Introduction}

Research on absorbers has become widespread to achieve enhanced absorption at selected wavelengths especially after Landy et al [1] first proposed achieving perfect absorption using meta-materials [2-14]. The first perfect absorber consisting of metallic split ring resonators and cutting wires was followed by many modifications to improve the band property [1]. However, most of the absorbers work at a single band frequency in limited wavelength spectrum. This restricted wavelength spectrum limits many practical applications such as multi-frequency spectroscopy and detection [15]. Moreover, the improvements in the performance of perfect absorbers usually results in complex and expensive fabrication methods which are significant bottlenecks for scalable implementation of perfect absorber technologies [16-18]. Thus, simple and high-performance absorbers that can operate in a wide spectral band without needing extra metallic patterns are desirable. In this work, based on theoretical models, we propose, and experimentally demonstrate perfect absorber surfaces based on silicon-on-insulator (SOI) wafers whose $\mathrm{Si}$ device layer and $\mathrm{Si}$ handle layer have appropriate carrier concentrations. Center wavelength of absorption of such absorber surfaces can be varied in infrared (IR) spectrum, and bandwidth of such absorber surfaces can be designed to be as large as $2.5 \mu \mathrm{m}$.

\section{Analytical approach}

The perfect absorber that we examine is a cavity layer of $\mathrm{SiO}_{2}$ that is sandwiched between two layers (i.e. device layer $\mathrm{Si}$, and handle layer Si of SOI wafers) of n-type Si. As can be seen in figure 1 , the top Si film layer has thickness of $d_{1}$, and index of refraction of $n_{1}$. The cavity layer has a thickness of $d_{2}$, and index of refraction of $n_{2} . t_{2}$ and $r_{1}$ should be noted as 


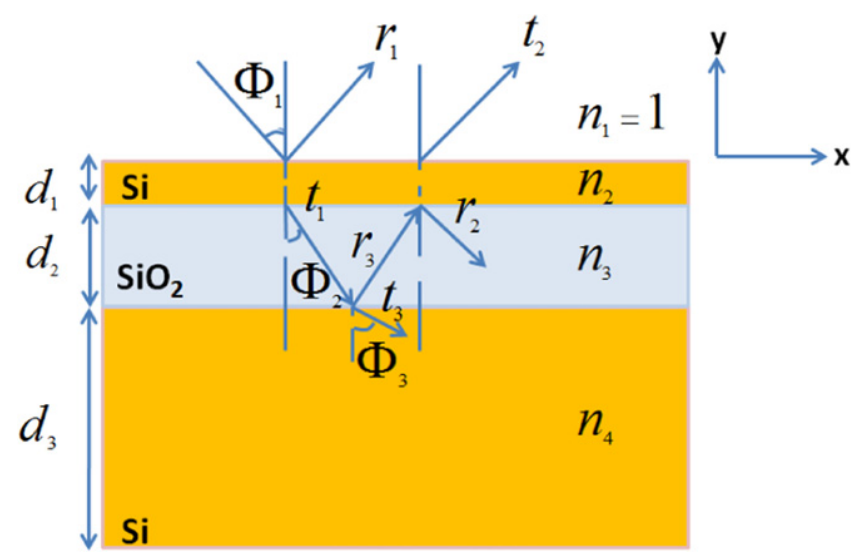

Figure 1. The schematic view of the absorber which is formed using a cavity layer of thickness $d_{2}$ sandwiched between a film of thickness $d_{1}$ and a semi-infinite opaque layer of thickness $d_{3}$. $t_{1}, t_{2}$ and $t_{3}$ are transmission coefficients; $r_{1}, r_{2}$ and $r_{3}$ are reflection coefficients at the corresponding film boundaries, respectively. In addition, these boundaries have corresponding angles of reflection and transmission, $\Phi_{1}, \Phi_{2}$ and $\Phi_{3}$, used to calculate transmittance and reflectance. In our case $\Phi_{1}=\Phi_{2}=\Phi_{3}=0$.

transmission and reflection coefficients at the air-Si device layer interface, respectively. $t_{1}$ and $r_{2}$ are transmission and reflection coefficients at the $\mathrm{SiO}_{2}$ and $\mathrm{Si}$ device layer interface, respectively. $t_{3}$ and $r_{3}$ are transmission and reflection coefficients at $\mathrm{SiO}_{2}$ and $\mathrm{Si}$ handle layer interface, respectively. With a thickness of $d_{3}$ and an index of refraction of $n_{4}$, the Si handle layer is thick enough (several hundred micrometers) that we can assume that it is semi-infinite. Analytical theories for optical response of structures are derived to be used in terms of thicknesses of layers, and complex refractive indices. The permittivity of $\mathrm{Si}$ is calculated according to the Drude model [19]. Furthermore, the transmission and reflection coefficients of the absorber can be stated as follows:

$$
\begin{gathered}
r=r_{1}+\frac{t_{1} t_{2} r_{3} \mathrm{e}^{-\mathrm{i} 2 \beta}}{1-r_{2} r_{3} \mathrm{e}^{\mathrm{i} 2 \beta}}, \\
t=\frac{t_{1} t_{3} \mathrm{e}^{\mathrm{i} 2 \beta}}{1-r_{2} r_{3} \mathrm{e}^{\mathrm{i} 2 \beta}},
\end{gathered}
$$

where $\beta=\frac{2 \pi n_{3} d_{3} \cos \varnothing_{2}}{\lambda}$ is the phase shift, $n_{3}$ is the refractive index of $\mathrm{SiO}_{2}, d_{2}$ is the thickness of $\mathrm{SiO}_{2}$ and $\varnothing_{2}$ is the angle of refraction at the interface of $\mathrm{Si}$ device layer, and $\mathrm{SiO}_{2}$. Reflectance and transmittance of the absorber can be calculated as follows:

$$
\begin{gathered}
R=r r^{*}, \\
T=\frac{\operatorname{Re}\left(\cos \varnothing_{3} / n_{3}\right)}{\operatorname{Re}\left(\cos \varnothing_{1} / n_{1}\right)} t t^{*} \text { for TM waves, } \\
T=\frac{\operatorname{Re}\left(\cos \varnothing_{3} n_{3}\right)}{\operatorname{Re}\left(\cos \varnothing_{1} n_{1}\right)} t t^{*} \text { for TE waves. }
\end{gathered}
$$

$\operatorname{Re}()$ indicates the real part of a complex quantity, the asterisk stands for the complex conjugate, and $n_{1}$ and $n_{2}$ indicate the complex refractive indices of air and $\mathrm{Si}$ device layer, respectively

$$
r r^{*}=\frac{\left.\left(p_{1}+p_{3} \delta\right)^{2} 4 p_{1} p_{3} \sin ^{2} \gamma_{1}\right)}{\left(1-p_{2} p_{3}\right)^{2}+4 p_{2} p_{3} \sin ^{2} \gamma_{2}}
$$

and

$$
t t^{*}=\frac{\alpha_{1} \alpha_{3}}{\left(1-p_{2} p_{3}\right)^{2}+4 p_{2} p_{3} \sin ^{2} \gamma_{2}},
$$

where $\rho=\left|r_{j}\right|$ and $\alpha=t_{j} t_{j}{ }^{*}$ with $j$ indicates 1,2 or 3 for the corresponding subscript, $\quad \delta=\left|t_{1} t_{2}-r_{1} r_{2}\right|$, $\gamma_{1}=\left(2 \beta-\varnothing_{1}+\varnothing_{3}+\varnothing_{2}\right) / 2, \quad \gamma_{2}=\left(2 \beta+\varnothing_{2}+\varnothing_{3}\right) / 2$, where $\varnothing_{j}=\arg \left(r_{j}\right)$ and $\varnothing_{2}=\arg \left(t_{1} t_{2}-r_{1} r_{2}\right)$.

In order to model the dielectric constant of $\mathrm{Si}$ that is expressed as a function of carrier mobility [19], the carrier mobility of n-type $\mathrm{Si}$ is calculated as

$$
\mu=88 T_{n}^{-0.57}+\frac{7.4 \times 10^{8} T^{-2.33}}{1+\left[\frac{N}{1.26 \times 10^{17} T^{2.4}}\right] 0.88 T_{n}^{-0.146}},
$$

where $T_{n}=T / 300, T$ is the temperature value in Kelvin, and $N$ is the carrier concentration in $\mathrm{cm}^{-3}$ [20]. In this study, $T$ is taken as $300 \mathrm{~K}$. When Drude model and equation (8) are used, optical parameters of Si for various carrier concentrations can be computed. Absorption, transmission and reflection are calculated utilizing Fresnel equations for conventional thinfilm wave propagation in absorbing media [21]. In our case, light is incident normal to the surface of the absorber. For the case of multiple interfaces, reflection and transmission coefficients can be obtained using Airy's formulae [22]. The reflectance and transmittance of the perfect absorber can be calculated by using equations (3)-(5). Since the Si handle layer is considered as semi-infinite, the absorptivity of the perfect absorber can be written as $\varepsilon_{\lambda, \phi}=1-R$ by Kirchhoff's law [20], where $R$ is reflectivity.

\section{Simulation}

The finite-difference-time-domain method was used to calculate the absorptivity of the perfect absorber. In simulations, a plane wave light spectrum of $2-25 \mu \mathrm{m}$ is normally incident to the absorber surface. The perfectly matched layer boundary condition at the bottom of the absorber is employed in order to model the semi-infinite Si handle wafer. Periodic boundary conditions were used for the left and right boundary conditions to model infinite structure in the $x$-direction. The minimum mesh size is set to $1 \mathrm{~nm}$. The reflected light power from the absorber is calculated by the field/power monitor above the structure. Reflected and absorbed power assumed to sum up to unity since transmission is zero. Power absorption is calculated using electric field intensity and the imaginary part of permittivity.

We varied the thickness of the n-Si device and cavity layers from 20 to $5000 \mathrm{~nm}$ for each layer, keeping the distance between the light source and top surface of the absorber constant. The carrier concentration of $\mathrm{n}$-Si handle layer was chosen at $10^{20} \mathrm{~cm}^{-3}$. However, carrier concentration of the $\mathrm{Si}$ device layer was varied between $10^{17}$ and $10^{20} \mathrm{~cm}^{-3}$. We 


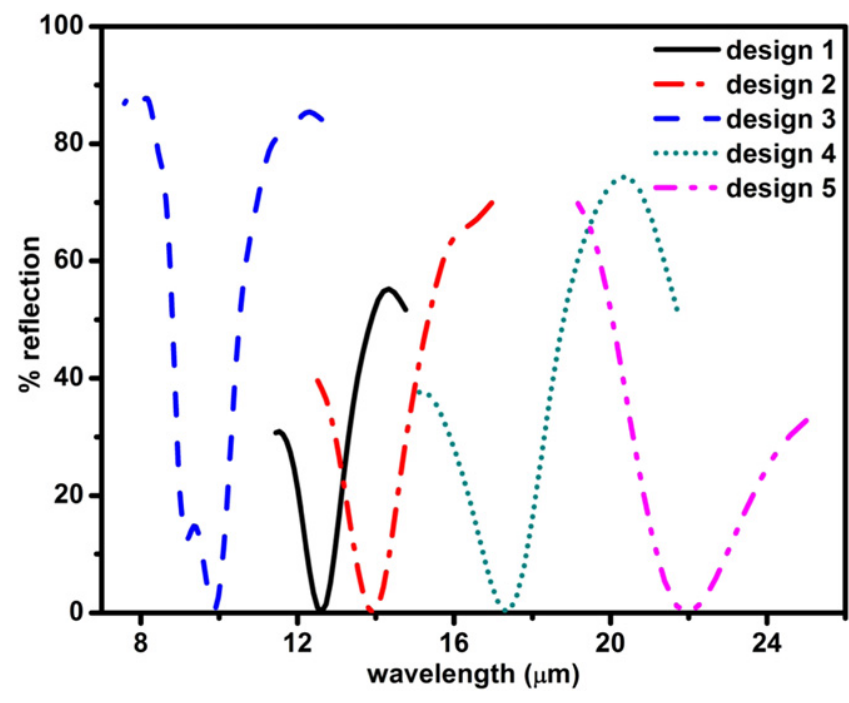

Figure 2. Zero-reflection-dips for five different designs. With altered perfect absorber designs we observe zero/near-zero reflection which is actually perfect absorption since transmission is zero. Perfect absorption is achieved in the IR region. For designs 1-5, center wavelengths of absorption are $12.6 \mu \mathrm{m}, 13.9 \mu \mathrm{m}, 9.8 \mu \mathrm{m}, 17.3 \mu \mathrm{m}$, $22 \mu \mathrm{m}$, respectively. For designs $1-5$, the parameters $\left(d_{1}, d_{2}\right.$, carrier concentration of Si device layer) are $(5000 \mathrm{~nm}, 5000 \mathrm{~nm}$,

$\left.10^{17} \mathrm{~cm}^{-3}\right),\left(5000 \mathrm{~nm}, 3000 \mathrm{~nm}, 10^{18} \mathrm{~cm}^{-3}\right),(4000 \mathrm{~nm}, 600 \mathrm{~nm}$, $\left.10^{17} \mathrm{~cm}^{-3}\right),\left(3000 \mathrm{~nm}, 4500 \mathrm{~nm}, 10^{17} \mathrm{~cm}^{-3}\right),(1500 \mathrm{~nm}, 3500 \mathrm{~nm}$, $\left.10^{18} \mathrm{~cm}^{-3}\right)$, respectively.

used n-Si for both layers instead of intrinsic Si to shift the plasma wavelength into the IR range.

\section{Results}

We classify our results as simulation results and experimental results that confirm our model.

\subsection{Simulation results}

In this study, appropriate carrier concentrations were chosen to find proper sets of refractive indices that will yield perfect or near perfect absorption by modifying the thicknesses of the layers $[23,24]$. The carrier concentrations of Si device layers were chosen on the order of $10^{18} \mathrm{~cm}^{-3}$ to provide refractive index contrasts with respect to the $\mathrm{Si}$ handle layer in the IR spectrum. Absorption mechanism of our designs are based on destructive interference of the reflected waves. If the waves destructively interfere at a single wavelength value, we observe single-band absorption. However, when the waves destructively interfere at multiple wavelength values, we are able to observe wide-band or multiple band absorption depending on how close the wavelength values are. Since refractive index of highly doped $\mathrm{Si}$ is strongly dependent on wavelength, it is possible to bring two or more values of resonance wavelength of perfect or near perfect absorption closer to each other. If many of resonance wavelength values are very close to each other, wideband absorption is observed, otherwise multiple band absorption is achieved. As demonstrated in figure 2, perfect absorption

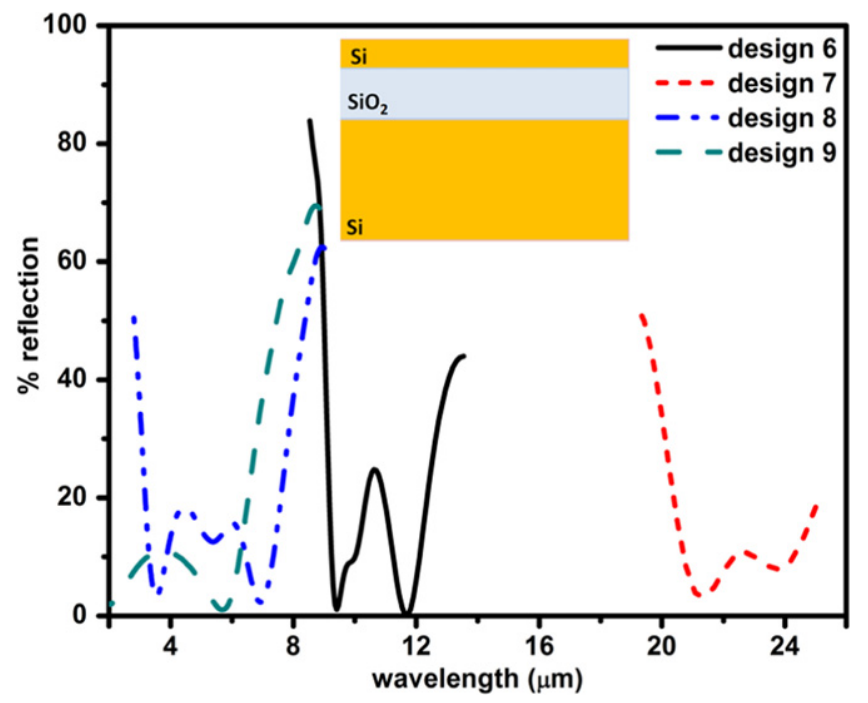

Figure 3. Multiple-band reflection dips for four different designs. We achieved multiple-band near-perfect absorbers in the IR region. For designs 6-9, center wavelengths of absorption are $10.7 \mu \mathrm{m}$, $22.4 \mu \mathrm{m}, 6.2 \mu \mathrm{m}, 4.1 \mu \mathrm{m}$, respectively. We achieved absorption bandwidths greater than $2 \mu \mathrm{m}$ where reflection is below $10 \%$. For designs 6-9, design parameters $\left(d_{1}, d_{2}\right.$, carrier concentration of $\mathrm{Si}$ device layer) are $\left(2500 \mathrm{~nm}, 4000 \mathrm{~nm}, 10^{17} \mathrm{~cm}^{-3}\right),(1000 \mathrm{~nm}$, $\left.5000 \mathrm{~nm}, 10^{17} \mathrm{~cm}^{-3}\right),\left(100 \mathrm{~nm}, 1500 \mathrm{~nm}, 10^{20} \mathrm{~cm}^{-3}\right),(100 \mathrm{~nm}$, $\left.100 \mathrm{~nm}, 10^{17} \mathrm{~cm}^{-3}\right)$, respectively.

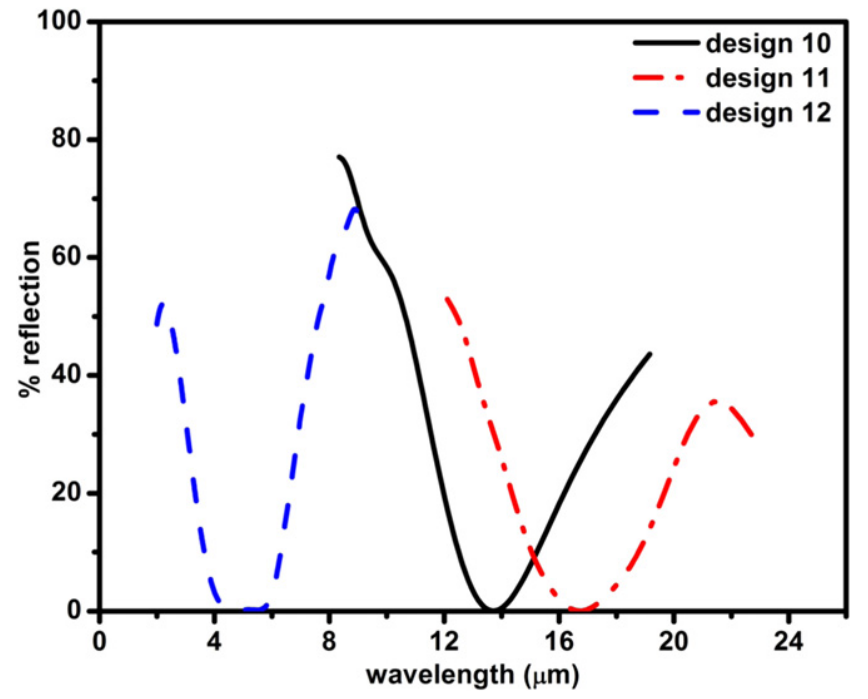

Figure 4. Wide-band reflection dips for three different designs. We obtain wideband zero-reflection, which is essentially wideband perfect absorption since transmission is zero. Wideband dips can be seen for various ranges of infrared spectrum. For design 10-12, $d_{1}$, $d_{2}$ and carrier concentration of $\mathrm{n}-\mathrm{Si}$ at the top are $1000 \mathrm{~nm}, 100 \mathrm{~nm}$, $10^{19} \mathrm{~cm}^{-3} ; 600 \mathrm{~nm}, 1500 \mathrm{~nm}, 10^{19} \mathrm{~cm}^{-3}$ and $60 \mathrm{~nm}, 600 \mathrm{~nm}$, $10^{20} \mathrm{~cm}^{-3}$, respectively. The carrier concentration of $\mathrm{Si}$ at the bottom is $10^{20} \mathrm{~cm}^{-3}$. Center wavelengths of design 10-12 are $13.6 \mu \mathrm{m}, 16.7 \mu \mathrm{m}$ and $5.4 \mu \mathrm{m}$, respectively. Bandwidths of absorption less than $10 \%$ of design $10-12$ are $2.6 \mu \mathrm{m}, 3.5 \mu \mathrm{m}$ and $2.7 \mu \mathrm{m}$, respectively.

is achieved in the wavelength range of $10-22 \mu \mathrm{m}$ with proper perfect absorber design. We have also observed multiple bands of perfect absorption in the IR range as can be seen in figure 3 . 


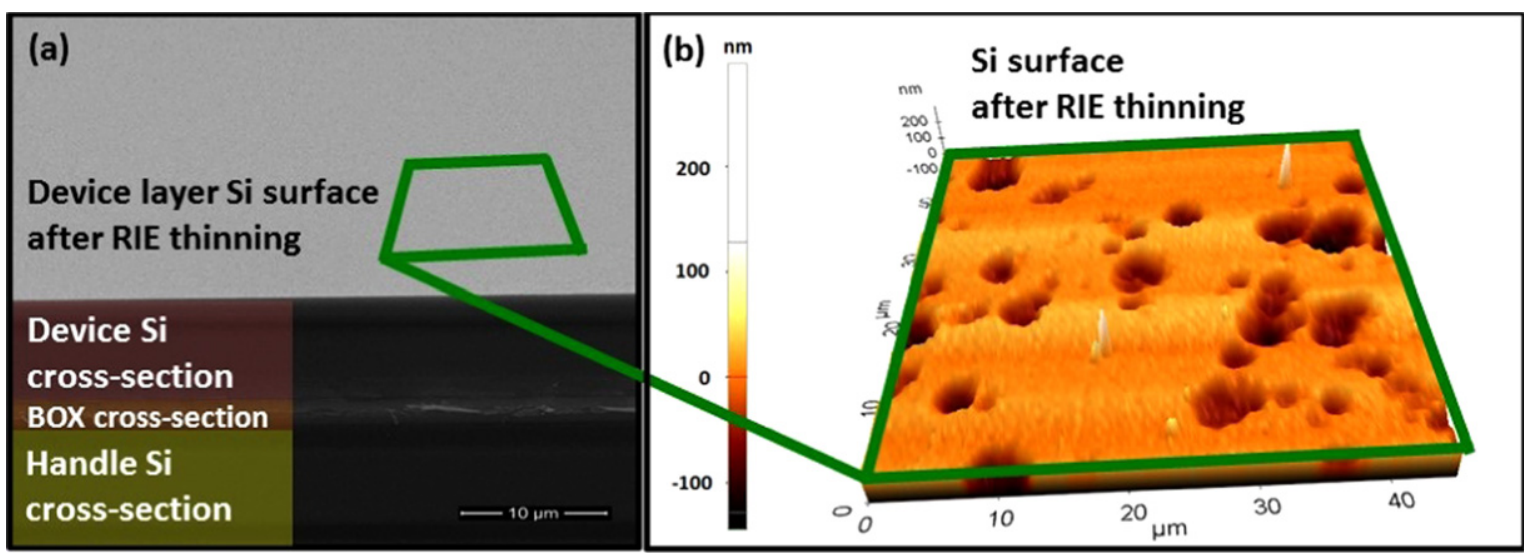

Figure 5. Cross-sectional SEM image and AFM scanned surface of thinned SOI wafer, respectively. In (a), from one of the RIE etched chips, we show that the final thickness of the device layer Si can be reduced to desired thickness values for each chip. In (b), the AFM image of the surface of the thinned SOI wafer is shown. Also, in (b) the image shows that surface roughness of the final Si surface is kept as smooth as possible using the $\mathrm{SF}_{6}$ based RIE recipe.

Comparing this work to that of Dayal et al [25], it is clear that our structure is significantly simpler. Moreover, in figure 4 we observe wide-band perfect absorption bands in the IR region. For design 11, there is a reflection dip (with average reflection below 6\%) with a bandwidth of almost $2.5 \mu \mathrm{m}$. For design 10, around wavelength of $14 \mu \mathrm{m}$, there is a dip of less than $6 \%$ with a spectrum range of almost $2 \mu \mathrm{m}$. And for design 12 , there is a reflection dip less than $2 \%$ whose wavelength range is almost $1.5 \mu \mathrm{m}$ in the wavelength range of $4-6 \mu \mathrm{m}$.

\subsection{Experimental results}

To experimentally confirm our models and simulations we followed the microfabrication approach below: the starting wafer is an SOI wafer with device layer resistivity in the range of $31.5-38.5 \Omega \mathrm{cm}$ and $\mathrm{Si}$ handle layer resistivity in the range of $3-5 \Omega \mathrm{cm}$. The thickness of $\mathrm{Si}$ handle layer is $575 \mu \mathrm{m}$. Orientation of the both Si layers is (100). Initially, device layer thickness of SOI is $54.5 \mu \mathrm{m}$ with $\pm 1.5 \mu \mathrm{m}$ variation as reported by the vendor. SOI wafer is cleaned with acetone, isopropyl alcohol, DI water, Piranha $\left(\mathrm{H}_{2} \mathrm{SO}_{4}: \mathrm{H}_{2} \mathrm{O}_{2}\right.$ 4:1), and HF dip to remove any residues that may be present on the device layer surface of the SOI wafer. Then, using an $\mathrm{SF}_{6}$ based reactive ion etching (RIE) plasma recipe, we isotropically thin the device layer $\mathrm{Si}$ of each sample to the desired $\mathrm{Si}$ thicknesses as shown in figure 5(a) for one of the experimentally tested devices. To measure the surface roughness of the Si device layer after RIE processing, atomic force microscopy is used to scan a $45 \mu \mathrm{m} \times 45 \mu \mathrm{m}$ area with a rate of $0.25 \mathrm{~Hz}$ using a cantilever whose spring constant is $0.2 \mathrm{~N} \mathrm{~m}^{-1}$. As shown in figure 5(b), an average of $36 \mathrm{~nm}$ surface roughness is achieved on the $\mathrm{Si}$ surface using an optimized RIE recipe. This surface roughness value is much smaller than our wavelength of interest, and satisfies the optical requirements for our experiments.

The thickness of the device layer $\mathrm{Si}$ is reduced to an effective thickness of $6.8 \mu \mathrm{m}$ with an average etch rate of $3 \mu \mathrm{m} \mathrm{min}^{-1}$. Then, we measure the reflection of an SOI sample between the $2-20 \mu \mathrm{m}$ wavelength ranges using

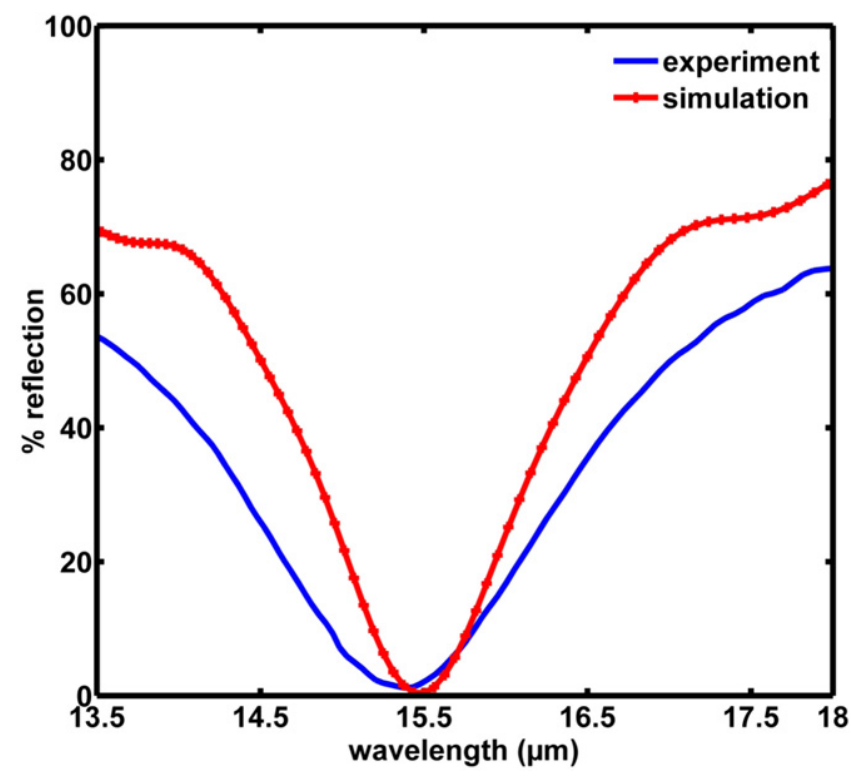

Figure 6. The theoretical and experimental reflection dips for a design whose effective device layer thickness is $6.8 \mu \mathrm{m}$. We observed an evident consistency between the theoretical and experimental optical responses of the design in IR wavelength spectrum, which is measured using FTIR.

Fourier transform infrared spectroscopy (FTIR). The simulation data and corresponding experimental data are shown in figure 6 indicating a good agreement between the simulation and experimental results. The experimental measurements are valid for light beams that are within $5^{\circ}$ uncertainty with respect to the surface normal. For higher deflection angles, the external reflection considerably reduces the optical power which penetrates into the absorber.

\section{Conclusion}

In this work, practical multi-featured perfect absorbers are designed and manufactured using $\mathrm{SiO}_{2}$ as a cavity layer that is 
sandwiched between an n-type Si device layer on top and an n-type Si handle layer at the bottom. When the carrier concentrations of the device and handle layers are chosen wisely with correspondingly appropriate thicknesses, we are able to achieve perfect absorbers whose resonance wavelengths vary between 2 and $25 \mu \mathrm{m}$. Moreover, multi-band perfect absorbers are observed for specific thicknesses and carrier concentrations of $\mathrm{Si}$ in the IR region.

We would like to emphasize four main achievements of this study that are reached by selecting the carrier concentration wisely, and the proper thicknesses for SOI layers: (i) we are able to obtain perfect absorbers whose resonance wavelengths vary between 2 and $25 \mu \mathrm{m}$. (ii) Multi-band perfect absorbers are observed in the IR region. (iii) $\mathrm{We}$ achieve perfect absorbers with greater than $98 \%$ absorption whose bandwidth is more than $1.5 \mu \mathrm{m}$. (iv) Starting from analytical formulations, we chose functional models, simulated the models, and eventually experimentally validated our models. In addition to these four main achievements above, it is also important to emphasize that the structural complexity, microfabrication costs, and implementation difficulty of the proposed perfect absorbers are significantly lower compared to the state of the art perfect absorbers. With the contribution of the four achievements above, this design is envisioned to be a crucial part in many future applications of detectors, sensing devices, meta-materials, modulators, micro-bolometers, sensors and antennas.

\section{Acknowledgments}

This work was supported by the Scientific and Technological Research Council of Turkey (TUBITAK), grant numbers 109E044, 112M004, 112E052 and 113M815. A.K.O. acknowledges support from the Turkish Academy of Sciences Distinguished Young Scientist Award (TUBA GEBIP).

\section{References}

[1] Landy N I, Sajuyigbe S, Mock J J, Smith D R and Padilla W J 2008 Perfect metamaterial absorber Phys. Rev. Lett. 100 207402

[2] Yan R H, Simes R J and Coldren L A 1989 Electroabsorptive Fabry-Perot reflection modulators with asymmetric mirrors IEEE Photonics Technol. Lett. 1 273-5

[3] Chin A and Chang T Y 1990 Multilayer reflectors by molecular-beam epitaxy for resonance enhanced absorption in thin high-speed detectors J.Vac. Sci. Technol. B $8339-42$

[4] Kishino K, Unlu M S, Jen-Inn C, Reed J, Arsenault L and Morkoc H 1991 Resonant cavity-enhanced (RCE) photodetectors IEEE J. Quantum Electron. 27 2025-34

[5] Yan R H, Simes R J and Coldren L 1991 Surface-normal electroabsorption reflection modulators using asymmetric Fabry-Perot structures IEEE J. Quantum Electron. 27 1922-31

[6] Law K K, Yan R H, Coldren L A and Merz J L 1990 Selfelectro-optic device based on a superlattice asymmetric Fabry-Perot modulator with an on/off ratio $\gtrsim 100: 1 \mathrm{Appl}$. Phys. Lett. 57 1345-7
[7] Tischler J R, Bradley M S and Bulović V 2006 Critically coupled resonators in vertical geometry using a planar mirror and a $5 \mathrm{~nm}$ thick absorbing film Opt. Lett. 31 $2045-7$

[8] Cui Y, Xu J, Hung Fung K, Jin Y, Kumar A, He S and Fang N X 2011 A thin film broadband absorber based on multi-sized nanoantennas Appl. Phys. Lett. 99253101

[9] Kats M A, Sharma D, Lin J, Genevet P, Blanchard R, Yang Z, Qazilbash M M, Basov D N, Ramanathan S and Capasso F 2012 Ultra-thin perfect absorber employing a tunable phase change material Appl. Phys. Lett. 101221101

[10] Zang X, Shi C, Chen L, Cai B, Zhu Y and Zhuang S 2015 Ultra-broadband terahertz absorption by exciting the orthogonal diffraction in dumbbell-shaped gratings Sci. Rep. 58901

[11] Cheng Y Z, Withayachumnankul W, Upadhyay A, Headland D, Nie Y, Gong R Z, Bhaskaran M, Sriram S and Abbott D 2015 Ultrabroadband plasmonic absorber for terahertz waves Adv. Opt. Mater. 3 376-80

[12] Cheng Y, Gong R and Cheng Z 2016 A photoexcited broadband switchable metamaterial absorber with polarization-insensitive and wide-angle absorption for terahertz waves Opt. Commun. 361 41-6

[13] Xu Y, Yuan W, Bie S, Xu H, Chen Q and Jiang J 2015 Broadband microwave absorption property of a thin metamaterial containing patterned magnetic sheet J. Electromagn. Waves Appl. 29 1-8

[14] Rhee J Y, Yoo Y J, Kim K W, Kim Y J and Lee Y P 2014 Metamaterial-based perfect absorbers J. Electromagn. Waves Appl. 28 1541-80

[15] Shu S and Yang L Y 2012 Metallic rugate structures for nearperfect absorbers in visible and near-infrared regions $O p t$. Lett. 37 3495-7

[16] Tao H, Bingham C M, Strikwerda A C, Pilon D, Shrekenhamer D, Landy N I, Fan K, Zhang X, Padilla W J and Averitt R D 2008 Highly flexible wide angle of incidence terahertz metamaterial absorber: design, fabrication, and characterization Phys. Rev. B 78241103

[17] Sun J, Liu L, Dong G and Zhou J 2011 An extremely broad band metamaterial absorber based on destructive interference Opt. Express 19 21155-62

[18] Grant J, Ma Y, Saha S, Khalid A and Cumming D R S 2011 Polarization insensitive, broadband terahertz metamaterial absorber Opt. Lett. 36 3476-8

[19] Cleary J W, Peale R E, Shelton D J, Boreman G D, Smith C W, Ishigami M, Soref R, Drehman A and Buchwald W R 2010 IR permittivities for silicides and doped silicon J. Opt. Soc. Am. B 27 730-4

[20] Arora N D, Hauser J R and Roulston D J 2002 Electron and hole mobilities in silicon as a function of concentration and temperature IEEE Trans. Electron Dev. 29 292-5

[21] Wang L P, Lee B J, Wang X J and Zhang Z M 2009 Spatial and temporal coherence of thermal radiation in asymmetric Fabry-Perot resonance cavities Int. J. Heat Mass Transfer 52 3024-31

[22] Cleary J W, Soref R and Hendrickson J R 2013 Long-wave infrared tunable thin-film perfect absorber utilizing highly doped silicon-on-sapphire Opt. Express 21 19363-74

[23] Zhang Z M 2007 Nano/Microscale Heat Transfer (New York: McGraw-Hill) ch 9

[24] Hums C, Finger T, Hempel T, Christen J, Dadgar A, Hoffmann A and Krost A 2007 Fabry-Perot effects in InGaN/GaN heterostructures on Si-substrate J. Appl. Phys. 101033113

[25] Govind D and Ramakrishna S A 2013 Design of multi-band metamaterial perfect absorbers with stacked metal-dielectric disks J. Opt. 15055106 